\title{
Dyslipidemia in stroke
}

\author{
Cynthia $\mathrm{A}^{1}$, Yogeesha KS ${ }^{2}$, Arunachalam $\mathrm{R}^{3}$ \\ ${ }^{1}$ Post-graduate resident, Department of General Medicine, Father Muller Medical College, Karnataka, India \\ ${ }^{2}$ Assistant Professor, Department of General Medicine, Father Muller Medical College, Karnataka, India \\ ${ }^{3}$ Professor, Department of General Medicine, Father Muller Medical College, Karnataka, India
}

\begin{abstract}
:
Aims and objects of study: To study the association of dyslipidemia with stroke.

Methods: Study design-Retrospective cross-sectional descriptive study Method of collection of data- A hundred patients between 30 to 90 years of age, with first ever stroke, during a 12-month period were included in the study. History and physical examination details were collected from patient records. Laboratory investigations were also obtained.Data Analysis- Data collected was analysed by frequency, mean, standard deviation and chi-square test.

Results: The lipid profile of the study sample was analysed according to the ATP III classification for identification of dyslipidemia. The findings revealed that 56\% of the patients had dyslipidemia. $40 \%$ had high total cholesterol, 7\% had high triglycerides and only 3\% had high LDL. However, 28\% of the patients showed low HDL levels. $10 \%$ had both high total cholesterol and low HDL.

Conclusion: This study showed a significant association of $56 \%$ between dyslipidemia and stroke. Although high LDL is usually held responsible for cerebrovascular accidents, our study showed a significant proportion of patients with low HDL. Thus, besides generating Indian evidence to correlate dyslipidemia with stroke, this study upholds that primary prevention is the key to overcoming the burden of stroke in our country.
\end{abstract}

Keywords: Dyslipidemia, Lipids and stroke, South India, Stroke

\section{Introduction}

Stroke is an illness of escalating socioeconomic importance, especially among the ageing population. According to the World Health Organization, stroke is the second leading cause of death world-wide. Thus, cerebrovascular disease is a huge public health problem imposing both a large disease burden and a large economic burden on our country. More disconcerting than this is the fact that this problem is accelerating with the passing years.

This is evident from the increasing prevalence and incidence of stroke in various studies. According to the Indian Council of Medical Research there were 930,985 cases of stroke in 2004 in India ${ }^{1}$ and the National Commission of Macroeconomics and Health has estimated that there will be 1.67 million stroke cases in India by $2015^{2}$.

Stroke is also a leading cause of morbidity, with $20 \%$ of survivors requiring institutional care after 3 months and $15-30 \%$ remaining permanently disabled ${ }^{3}$. Of all strokes, $88 \%$ are classified as ischemic, and the remainder $12 \%$ comprise of hemorrhagic, either subarachnoid $(9 \%)$ or intracerebral $(3 \%){ }^{4}$.

Dyslipidemia has been a known major risk factor for coronary heart disease. However more recently, it has been established as a risk factor in cerebrovascular disease ${ }^{5}$. Elevated low density lipoprotein cholesterol (LDL-C) is a risk factor for coronary artery disease whereas high levels of high-density lipoprotein cholesterol (HDL-C) are protective ${ }^{6-10}$. However, the role of lipids as risk factors for ischemic stroke has been less consistently observed.

The discordant results of observational studies may be attributed to the heterogenecity of stroke, since dyslipidemia is less likely to play a major role in the pathogenesis of some ischemic stroke subtypes (e.g. lacunar and cardioembolic strokes) and elevated LDL-C levels might increase the risk of hemorrhagic stroke. In view of this ambiguity in the relationship between lipids and stroke, this study was carried out to ascertain the association between dyslipidemia and stroke in patients who presented to our hospital .

2.1 Objective of the study:

\section{Subjects and Methods}

To explore the relationship between lipid profile components and incident ischemic stroke in a first - ever stroke cohort. 


\subsection{Materials and methods} Source of data:

A detailed history and physical examination details were collected from patient records in the hospital medical record department. Laboratory investigations (fasting lipid profile) were also obtained from the records. Method of collection of data:

A total of hundred patient records were accessed from the medical records department in Father Muller medical college hospital.

Design of the study:

Retrospective cross-sectional descriptive study

Duration of the study:

The study was carried out on patients presenting with stroke during a 12-month period from $1^{\text {ST }}$ January 2012 to 31st December 2012 .

\section{Inclusion Criteria}

A hundred patients between 30 to 90 years of age who were admitted by the Department of General Medicine, Father Muller Medical College with first ever stroke, verified by CT scan brain during a 12-month period from January 1st 2012 to December 31st 2012 were included in the study.

\section{Exclusion Criteria:}

Patients who had a recent myocardial infarction with left ventricular failure, history of syncopal attacks, neurological deficits secondary to epilepsy, infective or metastatic disorder, or pre-existing severe cognitive disorder were excluded from this study.

\section{Data Analysis}

Data collected was analysed by frequency, mean, standard deviation and chi-square test.

\section{Results:}

A total of 100 patients were included in this study. Detailed history and physical examination details were obtained from the medical records section. All the patients included in the study were patients with first ever stroke verified by CT scan. Among them, $66 \%$ were males and $34 \%$ were females. The number of patients with stroke increased as the age group increased. Thirty-four percent of the patients were between 60-69 years of age and $28 \%$ were between $70-79$ years of age. Thus, over $62 \%$ of them were above the age of 60 yrs at the time of presentation.

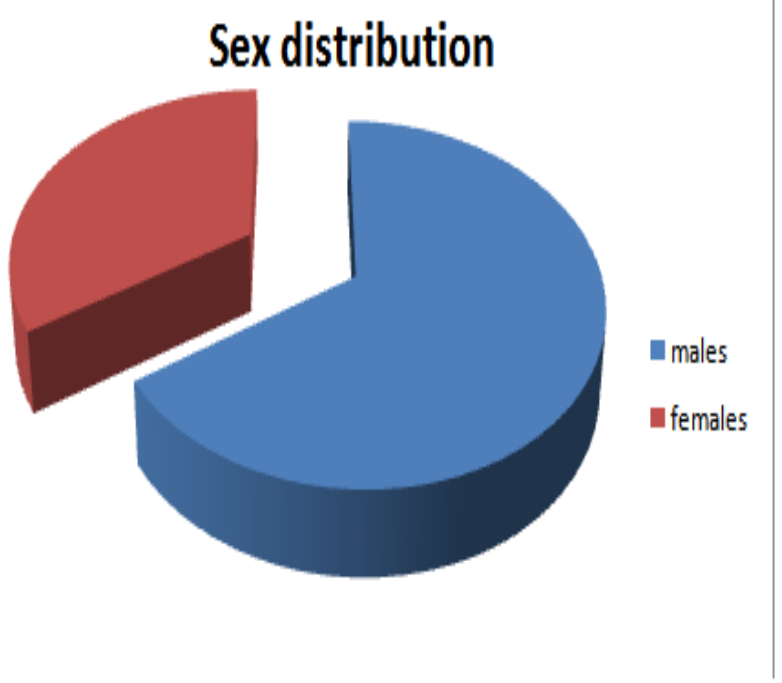

Fig. 1- Sex distribution among the study population 


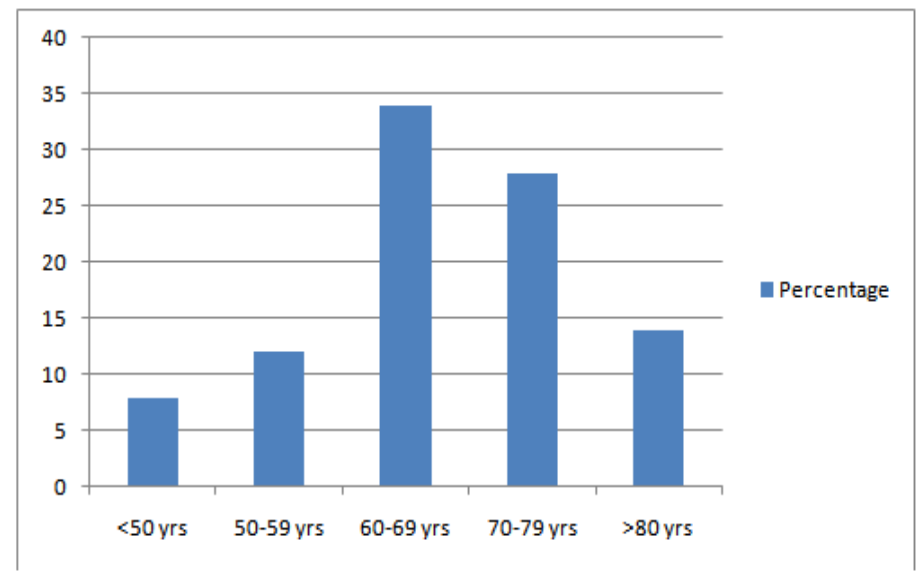

Fig. 2- Age distribution among the study population

The classical risk factors hypertension and diabetes mellitus were also studied. The data showed that $55 \%$ were hypertensive and $30 \%$ were diabetic. While $24 \%$ of the study sample had both hypertension and diabetes, $39 \%$ of the patients had none of these.

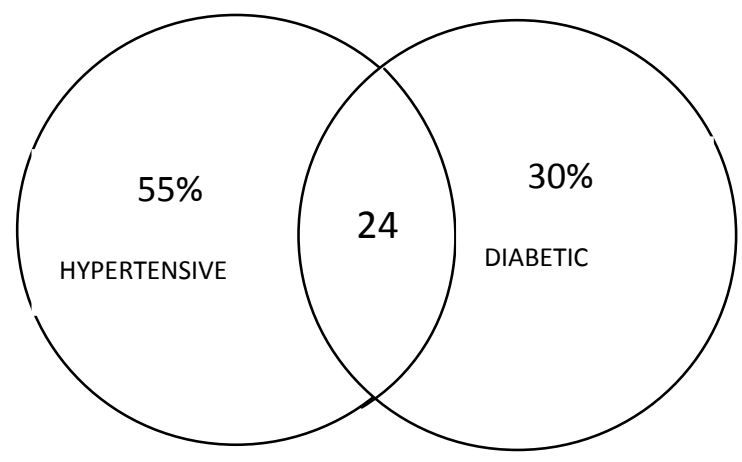

Fig. 3- Risk factors

The lipid profile of the study sample was analysed according to the ATP III classification for identification of dyslipidemia. The findings revealed that $56 \%$ of the patients had dyslipidemia, thereby proving that a majority of the study population had abnormal baseline lipid profiles. Among the dyslipidemic patients, $40 \%$ had elevated total cholesterol, $7 \%$ had elevated triglycerides and only 3\% had elevated LDL. However, interestingly $28 \%$ of the patients showed low HDL levels. Ten percent of the patients had both high total cholesterol and low HDL.

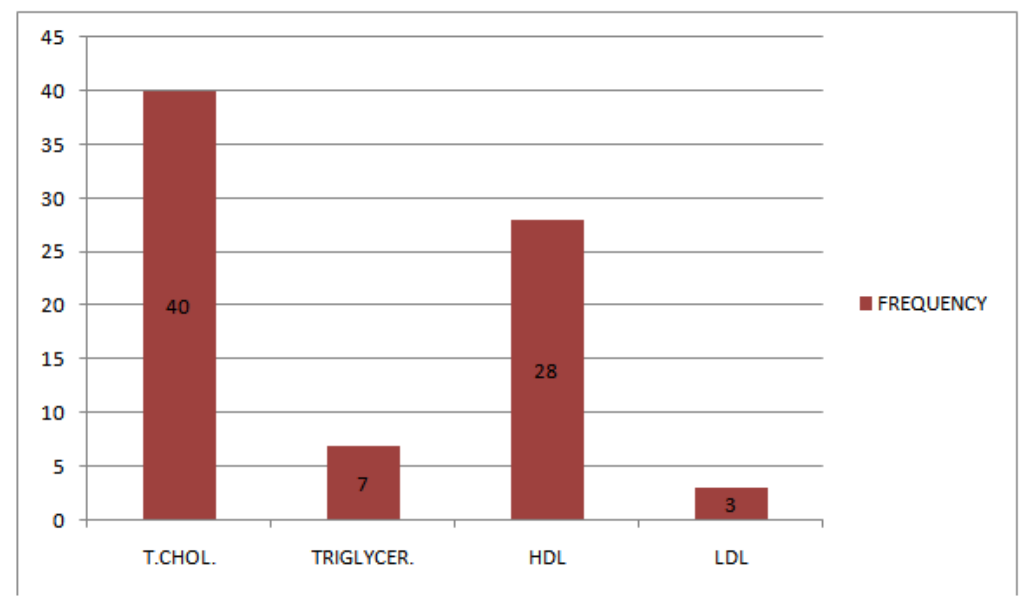

Fig. 4: Lipid profile among stroke patients

IV. Discussion: 
Ischemic stroke, which is the most commonly occuring cerebrovascular accident, is mostly due to thromboembolism secondary to atherosclerosis in the major arteries. Nikolai first proposed a link between cholesterol and atherosclerosis in 1912 . In a research, oft cited as a breakthrough research in atherosclerosis in the $20^{\text {th }}$ century, Nikolai and his team proved the obstructive pathophysiology in atherosclerosis occurs as a result of increased cholesterol levels. ${ }^{14}$

However, though there exist several risk factors for cerebrovascular disease, atherosclerosis is recognized as one of the leading causes of brain ischemia.

Although risk factors like age, sex, race and gender are non-modifiable factors causing stroke, in this study we will analyse the pattern of the modifiable risk factor, lipids in patients with stroke.

Our study showed a preponderance of males among the study population thus reflecting an overall male sex presdisposition to stroke. The total males under study were $66 \%$ while the females under study were $34 \%$. This corroborated with a study done on lipid profile in stroke in Bahrain which also showed an increased incidence in stroke among the males when compared to females. ${ }^{15}$

An age distribution among the study population was also analysed and clustering of cases was found between 60-69 years of age. A majority of the study population was above the age of 50 years., thereby affirming the increasing trend of stroke with age. This also correlates with data obtained from a study on lipid profile done on stroke patients in Northern Manhattan where the mean age was $68.8 \%{ }^{16}$

Moving further east, a study done on lipid profile in Pakistan also showed an increased frequency of patients $(28 \%)$ figuring within the 61-70 age group. ${ }^{17}$

This study also analysed the other risk factors frequently encountered in stroke such as hypertension and diabetes. $55 \%$ of the patients were found to be hypertensive while $30 \%$ were diabetic. Both hypertension and diabetes were noted in $24 \%$ of the population. On comparing these results to the lipid profile study done in Bahrain, hypertension was found to be the consistent major risk factor in the occurence of stroke. However, in contrast to this study where only $10.5 \%$ of the population showed no risk factors, our study showed that $39 \%$ of the study population was neither diabetic nor hypertensive. ${ }^{15}$

The core of this study revolved around identification of an association between dyslipidemia and ischemic cerebrovacular accident. The percentage of dyslipidemic individuals among the study population amounted to $56 \%$, thus showing a positive correlation between dyslipidemia and stroke among patients in the South Indian population under study. The pattern of dyslipidemia showed that $40 \%$ of the patients with abnormal lipid profiles had an elevated serum total cholesterol while only $7 \%$ had elevated triglycerides. Surprisingly, only $3 \%$ of the dyslipidemic patients had elevated LDL which is usually implicated in atherosclerosis while $28 \%$ of the individuals had low HDL, thereby showing a unique correlation between stroke and low HDL levels.

When this data was compared to the data obtained from other studies done on lipid profile in stroke, it was found that the results do not correlate. The study on dyslipidemia in stroke patients done in Pakistan, showed that $32 \%$ of the study population had high total cholesterol and $24 \%$ had high triglycerides. Though the serum total cholesterol frequency among both the population correlate, our study population in coastal South India showed only a small percentage of patients with hypertriglyceridemia (7\%) in comparison to the study done in Pakistan ${ }^{17}$ where $24 \%$ of the study population had hypertriglyceridemia. This may be attributed to the protective effect of frequent fish consumption among this western coastal belt of South India. This theory is also supported by findings from a meta-analysis done on several cohort studies that proved the inverse relation of fish intake to risk of stroke. ${ }^{18}$

Further, our study showed that only $3 \%$ of the dyslipidemic patients had elevated LDL in contrast to $26 \%$ of the patients having elevated LDL in the Pakistani study. Low HDL levels were also more frequently implicated among our stroke patients $(28 \%)$ than in the Pakistani study population $(18 \%)$.

\section{Conclusion:}

This study showed an association of $56 \%$ between dyslipidemia and stroke.These findings are consistent with other similar studies done in Bahrain, Manhattan and Pakistan. They show a definite relationship between dyslipidemia and stroke. The occurrence of the underlying co-morbities of hypertension and diabetes also corroborated with the above mentioned studies.

Although high LDL is usually held responsible for genesis of cerebrovascular accidents, our study showed a significant proportion of patients with low HDL. This validates the importance of dietary and lifestyle modifications to combat this crippling disease. Thus, besides generating Indian evidence to correlate dyslipidemia with stroke, this study upholds that primary prevention is the key to overcoming the burden of stroke in our country. 


\section{References:}

[1]. Central Bureau of Health Intelligence. National Health Profile. New Delhi: Directorate General of Health Sciences, 2008:102

[2]. World Health Organization. Workshop Report on Stroke Surveillance in India. (last checked 28 June 2012)

[3]. Gotto AM. Some reflections on arteriosclerosis: past, present, and future. Circulation 1985; 72:8-17.

[4]. American Heart Association. Heart disease and stroke statistics-2006 update: A report from the American Heart Association Statistics Committee and Stroke Statistics Committee. Circulation 2006 e85-e151.

[5]. Goldstein LB, Adams R, Becker K, Furberg CD, Gorelick PB, Hademenos G et al. Primary Prevention of Ischemic Stroke: A Statement for Healthcare Professionals from the Stroke Council of the American Heart Association. Circulation. 2001; 103:163.

[6]. Ballantyne CM, Herd JA, Ferlic LL, et al. Influence of low HDL on progression of coronary artery disease and response to fluvastatin therapy. Circulation. 1999; 99(6):736-743.

[7]. Gordon DJ, Probstfield JL, Garrison RJ, et al. High-density lipoprotein cholesterol and cardiovascular disease: four prospective American studies. Circulation.1989;79(1):8-15.

[8]. Miller GJ, Miller NE. Plasma-high-density-lipoprotein concentration and development of ischaemic heart-disease. Lancet. 1975;1(7897):16-19.

[9]. Stampfer MJ, Sacks FM, Salvini S, Willett WC, Hennekens CH. A prospective study of cholesterol, apolipoproteins, and the risk of myocardial infarction. N Engl J Med. 1991;325(6):373-381.

[10]. Yaari S, Goldbourt, Even-Zohar S, Neufeld HN. Associations of serum high density lipoprotein and total cholesterol with total, cardiovascular, and cancer mortality in a 7-year prospective study of 10000 men. Lancet. 1981;1(8228):1011-1015.

[11]. Iso H, Jacobs DR , Wentworth D, et al. Serum cholesterol levels and six-year mortality from stroke in 350,977 men screened for the Multiple Risk Factor Intervention Trial. N Engl J Med. 1989; 320:904-10.

[12]. Zhang X, Patel A, Horibe H, Wu Z, Barzi F, Rodgers A, et al; Asia Pacific Cohort Studies Collaboration. Cholesterol, coronary heart disease, and stroke in the Asia Pacific region. Int J Epidemio. 2003; 32: 563-72.

[13]. Horenstein RB, Smith DE, Mosca L. Cholesterol predicts stroke mortality in the Women's Pooling Project. Stroke. 2002; 33: 18638 .

[14]. Konstantinov IE, Mejevoi N, Anichkov NM. Tex Heart Inst J. 2006; 33(4): 417-423.

[15]. Al-Jishi AA, Pochiraju KM. Profile of stroke in Bahrain. Neurosiences 2000; Vol. 5 (1): 30-34.

[16]. Willey JZ; Qiang X, Albala BB, Myunghee CP, Moon YP, Sacco RL, Elkind MSV. Lipid profile components and risk of stroke-A Northern Manhattan study. Arch Neurol. 2009;66(11):1400-1406.

[17]. Baluch UT, Kiani I, Zaib-un-nisa ,Badshah M. Association of dyslipidemia and ischemic stroke. Ann. Pak. Inst. Med. Sci. 2008;4(3): 165-167.

[18]. Ka He, Song Y, Daviglus ML, Liu K, Horn LV, Dyer AR et al. Fish consumption and incidence of stroke. Stroke.2004:35:153842. 